\title{
Nandinine, a Derivative of Berberine, Inhibits Inflammation and Reduces Insulin Resistance in Adipocytes via Regulation of AMP-Kinase Activity
}

\author{
Authors \\ Wenwen Zhao ${ }^{1,2}$, Haixia $\mathrm{Ge}^{3}$, Kang Liu ${ }^{1}$, Xiuping Chen ${ }^{2}$, jian Zhang ${ }^{4}$. \\ Baolin Liu ${ }^{1}$ \\ Affiliations \\ 1 Department of Pharmacology of Chinese Materia Medica, China \\ Pharmaceutical University, Nanjing, P. R. China \\ 2 State Key Laboratory of Quality Research in Chinese Medicine, \\ Institute of Chinese Medical Sciences, University of Macau, Macao, \\ P. R. China \\ 3 Department of Pharmacy, Huzhou University, Huzhou, P. R. China \\ 4 State Key Laboratory of Natural Medicines, China Pharmaceutical \\ University, Nanjing, P. R. China
}

Key words

Berberine, nandinine, insulin resistance, 3T3-L1 adipocytes,

Inflammation, AMPK

received April 23, 2016

revised May 30, 2016

accepted June 11, 2016

\section{Bibliography}

DOI http://dx.doi.org/10.1055/s-0042-110576

Published online July 27, 2016 | Planta Med 2017; 83: 203-209

(c) Georg Thieme Verlag KG Stuttgart · New York I ISSN 0032-0943

Correspondence

Prof. Baolin Liu

Department of Pharmacology of Chinese Materia Medica, China

Pharmaceutical University
639 Longmian Road, Nanjing, 211198, P. R. China

Phone: + 862586185127 , Fax: + 862586185292

zhongyao440@163.com

\section{ABSTRACT}

Nandinine is a derivative of berberine that has high efficacy for treating cardiovascular diseases. This study investigated the effects of berberine and nandinine on the regulation of insulin sensitivity in adipocytes. Through treatment with macrophage-derived conditioned medium in 3T3-L1 adipocytes, dysregulation of adipokine production and activation of the IKB kinase $\beta /$ nuclear factor-kappa B pathway was induced. However, these phenomena were effectively reversed by berberine, nandinine, and salicylate pretreatments. Furthermore, both berberine and nandinine inhibited serine phosphorylation of insulin receptor substrate- 1 induced by IкB kinase $\beta$ and increased tyrosine phosphorylation of insulin receptor substrate- 1 to activate the PI3K/Akt pathway, which finally led to insulin-mediated glucose uptake. In addition, berberine and nandinine significantly increased AMP-activated protein kinase activity, thereby contributing to their anti-inflammatory effect by inhibiting I $\mathrm{KB}$ kinase $\beta$ activation. Finally, in vivo studies demonstrated that both berberine (100 or $200 \mathrm{mg} / \mathrm{kg}$ ) and nandinine (100 or $200 \mathrm{mg} / \mathrm{kg}$ ) effectively ameliorated glucose intolerance and induced the insulin sensitivity index in mice. In conclusion, berberine and nandinine attenuated insulin resistance in adipocytes by inhibiting inflammation in an AMP-activated protein kinase-dependent manner. Berberine and nandinine may be used as dietary supplements and nandinine is a new candidate for obesity treatment.

\section{Introduction}

Obesity, characterized as a state of low-level inflammation, is associated with insulin resistance [1,2]. In adipose tissue of obese patients, recruited macrophages can activate the inflammatory response in neighboring adipocytes by releasing proinflammatory cytokines such as TNF- $\alpha$ and interleukin-6 (IL-6) [3-5]. These inflammatory molecules impair insulin receptor substrate-1 (IRS-1) function and downstream insulin/PI3K signaling to block glucose uptake, thereby leading to insulin resistance in adipocytes [6]. In addition, AMP-activated protein kinase (AMPK) regulates glucose and lipid homeostasis [7]. Emerging evidence demonstrates that anti-inflammatory action of AMPK is implicated in the reduction of insulin resistance $[8,9]$.

Berberine is a major isoquinoline alkaloid present in the Chinese herb Rhizoma coptidis and has a wide range of pharmacological actions $[10,11]$. Recently, its antidiabetic action and related mechanisms have led to an increased interest among people. Due to the low bioavailability of berberine in vivo [12], a higher number of derivatives have been developed [13]. Nandinine is a benzylisoquinoline alkaloid derived from berberine, and this derivative contains an unsaturated heterocycle ring in the skeleton and a hydroxyl group instead of the methoxyl group in the C-9 position as compared to berberine. Although it was first isolated more than 80 years ago, few studies reporting its pharmacological effect have been published. In this study, we found that both berberine and nandinine attenuated insulin resistance by inhibiting inflammation in an AMPK-dependent manner in vivo and in vitro. A parallel investigation would present new insights on the development of derivatives from berberine for the management of diabetes and insulin resistance. 


\section{Results}

In the present study, the production of TNF- $\alpha$ and IL-6 was increased after macrophage-derived conditioned medium (MacCM) treatment in 3T3-L1 adipocytes. Pretreatment with berberine and nandinine ( $\bullet$ Fig. $\mathbf{1}$ ) effectively reversed these phenomena ( $\vee$ Fig. 2A-D). Adiponectin is a novel adipocyte-specific protein and an anti-inflammatory factor [14]. Mac-CM showed a reduction in adiponectin secretion. However, berberine and nandinine pretreatment obviously restored the production of adiponectin ( $\bullet$ Fig. 2E, F). Salicylate, a positive control, also showed activities similar to those of berberine and nandinine.

Dysregulated secretion of adipokines is the response to inflammation [15], and I $\mathrm{KB}$ kinase $\beta$ (IKK $\beta$ ) could modulate proinflammatory cytokine expression by activating nuclear factor-kappa B (NF$\kappa B)$ in many types of cells [16-18]. In the present research, stimulation of Mac-CM enhanced the phosphorylation of IKK $\beta$ $\left(\vee\right.$ Fig. 3A), the interaction of $I_{\kappa} \mathrm{B} \alpha$ and $\operatorname{IKK} \beta$ ( $\vee$ Fig. $\left.3 \mathrm{~B}, \mathrm{C}\right)$, and the translocation of activated NF- $K B$ into the nucleus ( $\vee$ Fig. $3 D$ ), whereas these phenomena were reversed by berberine, nandinine, and salicylate ( $\triangleright$ Fig. 3 ).

IRS-1 is the main link between inflammation and insulin resistance [9]. After serine phosphorylation (S307) of IRS-1 is activated by IKK $\beta$, the reduced tyrosine phosphorylation (Tyr) of IRS-1 will impair the insulin pathway to block glucose uptake in adipocytes $[19,20]$. In this study, serine phosphorylation (S307) of IRS-1 was activated by Mac-CM-induced IKK $\beta$ ( $\triangleright$ Fig. $4 \mathrm{~A}$ ). Meanwhile, the reduction of the tyrosine phosphorylation (Tyr) of IRS-1 ( $\vee$ Fig. 4B) significantly decreased PI3K and Akt phosphorylation ( Fig. 4C,D), and finally blocked insulin-stimulated glucose transporter-4 (GLUT-4) translocation and glucose uptake ( $\vee$ Fig. 4E, F). Berberine, nandinine, and salicylate restored tyrosine phosphorylation to activate the insulin pathway, and stimulated GLUT-4 translocation as well as glucose uptake in the presence of insulin in adipocytes.

Activation of AMPK improves inflammation and insulin resistance in adipose tissues [21]. Results from our study showed that berberine, nandinine, and the AMPK activator 5-aminoimidazole4-carboxamide ribonucleotide (AICAR) increased AMPK activity in both normal and Mac-CM-induced adipocytes ( $\bullet$ Fig. 5 A, B). Furthermore, activated AMPK effectively inhibited IKK $\beta$ activation $(\vee$ Fig. 5C) to restore PI3 K expression ( $\vee$ Fig. 5D) and GLUT-4 translocation ( $\triangleright$ Fig. 5E, F), which were impaired by Mac-CM. All of the beneficial effects from berberine, nandinine, and AICAR could be obviously abolished by AMPK inhibitor compound C pretreatment. These results suggested that AMPK was involved in the regulation of insulin resistance in adipocytes.

Finally, we investigated the influence of berberine and nandinine on glucose tolerance under inflammatory conditions in vivo. As an inflammatory challenge, Mac-CM treatment induced glucose intolerance in mice as evidenced by reduced glucose disposal. Similar to salicylate, the oral administration of berberine and nandinine (from $100 \mathrm{mg} / \mathrm{kg}$ to $200 \mathrm{mg} / \mathrm{kg}$ ) obviously restored glucose disposal in the presence of Mac-CM ( $\bullet$ Fig. 6A). An increase of total glucose under AUC (AUC-G) was also reduced by both berberine and nandinine treatment ( $\bullet$ Fig. 6 B). In addition,

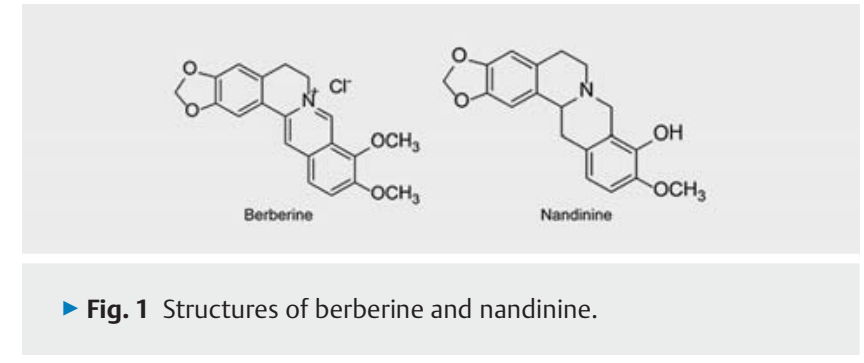

Mac-CM-induced insulin resistance was also effectively attenuated by berberine and nandinine treatment as evidenced by the decreased homeostasis model assessment of the insulin resistance (HOMA-IR) index ( $\bullet$ Fig. 6C).

\section{Discussion}

Inflammation is involved in the initiation and development of insulin resistance $[22,23]$. Many inflammatory molecules such as TNF- $\alpha$ and free fatty acids could induce insulin resistance in many different cell types $[13,24]$. AMPK is a metabolic sensor that helps maintain cellular energy homeostasis and it can exert significant anti-inflammatory effects in vitro [25-27]. In the present study, we showed that both berberine and nandinine reduced insulin resistance in an AMPK-dependent manner in adipocytes.

Mac-CM was derived from activated macrophages rich in inflammatory mediators, including TNF- $\alpha$ and IL-6, which can better mimic the pathology of insulin resistance in diabetes and obesity. In our study, Mac-CM stimulation obviously evoked inflammation in adipocytes, as evidenced by enhanced IKK $\beta$ phosphorylation and elevated levels of TNF- $\alpha$ and IL-6. However, these phenomena were reversed by berberine and nandinine. Besides, adiponectin is a novel adipocyte-specific protein and plays a role in the development of insulin resistance [28]. Berberine and nandinine also effectively increased adiponectin production, which was reduced by Mac-CM in adipocytes. Therefore, the beneficial effects of berberine and nandinine on the regulation of inflammatory cytokine well demonstrated their anti-inflammatory potency.

IRS-1 is a key control linking inflammation to insulin resistance [24]. Increased serine phosphorylation (S307) of IRS-1 could disturb Tyr of IRS-1, thereby leading to the impairment of the insulin signaling pathway [29]. Besides, IKK $\beta$ is required for activating serine phosphorylation of IRS-1 [30]. In the study, as expected, Mac-CM inhibited tyrosine phosphorylation of IRS-1 mediated by increased IKK $\beta$ to impair the PI3K/Akt pathway and finally blocked glucose uptake. Berberine and nandinine effectively attenuated insulin resistance by targeting IKK $\beta$.

AMPK is a crucial regulator of energy metabolism and controls many characteristics of cellular stress resistance [31]. Accumulated evidence demonstrated that AMPK has a close relationship with insulin resistance [32]. Berberine reportedly suppresses proinflammatory responses through AMPK activation in macrophages [33]. Hence, we investigated whether berberine and nandinine regulated insulin signaling in an AMPK-dependent manner in 3T3-L1 adipocytes. In our study, both berberine and nandinine 


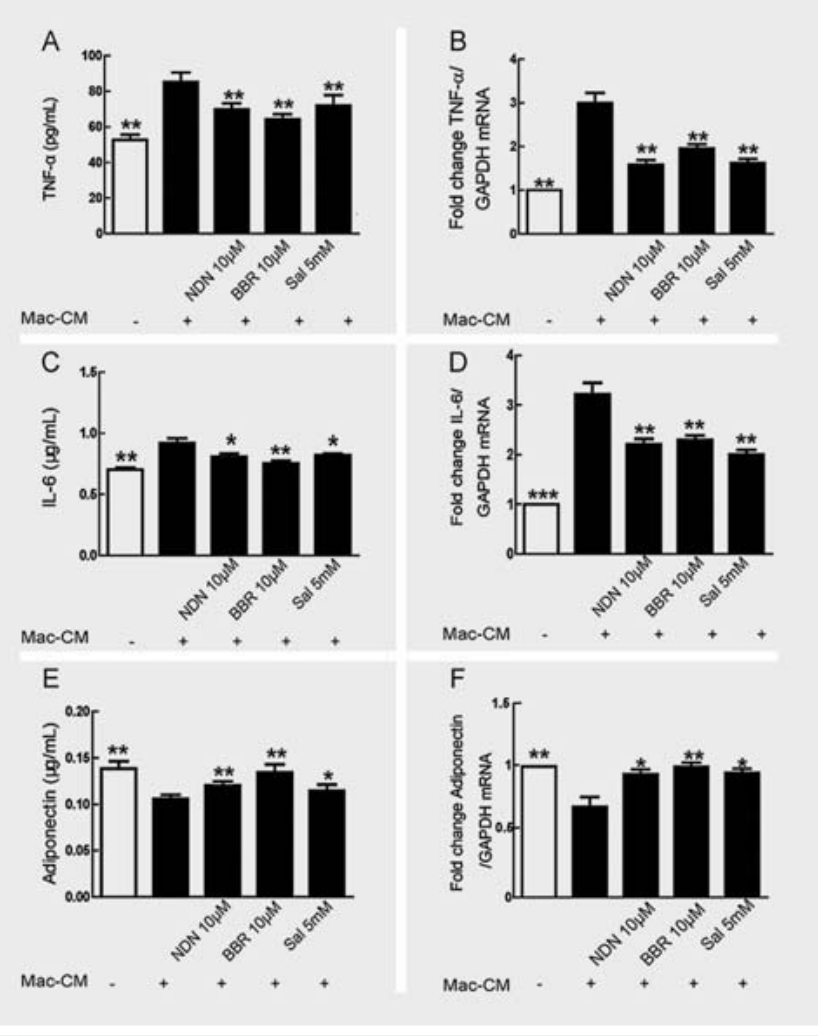

- Fig. 2 Berberine and nandinine regulated the dysregulated secretion of adipokines induced by Mac-CM in adipocytes. Cells were pretreated with nandinine $(10 \mu \mathrm{M})$, berberine $(10 \mu \mathrm{M})$, or salicylate $(5 \mathrm{mM})$ for $1 \mathrm{~h}$ and then incubated with Mac-CM for $24 \mathrm{~h}$. Amounts of the cytokines in the culture media were quantified by ELISA ( $A, C$, and $\mathrm{E}$ ) and RT-PCR (B, D, and F). Data are presented as the mean \pm $\mathrm{SD}$ of three independent experiments. ${ }^{*} P<0.05$ vs. Mac-CM, ${ }^{* *} \mathrm{p}<0.01$ vs. Mac-CM, ${ }^{* * *} \mathrm{p}<0.001$ vs. Mac-CM. Mac-CM, group with Mac-CM treatment alone; NDN, nandinine; BBR, berberine; Sal, salicylate.

increased AMPK activation in normal and Mac-CM-damaged adipocytes. Additionally, berberine, nandinine, and AICAR (AMPK activator) effectively reversed Mac-CM-induced phosphorylation of IKK $\beta$ to restore PI3 K signaling, thereby leading to GLUT-4 translocation onto membranes and glucose uptake in the presence of insulin. These results suggested that berberine and nandinine ameliorated insulin resistance by activating AMPK.

To clarify the effects of berberine and nandinine on the actions of insulin in vivo, we detected the effects of them on glucose tolerance in mice. Glucose load stimulates insulin secretion from pancreatic islets, and then insulin, in turn, promotes glucose uptake [34]. In our study, the administration of berberine and nandinine effectively ameliorated glucose intolerance and induced the insulin sensitivity index in mice, thereby hinting that berberine and nandinine ameliorated glucose intolerance by restoring insulin sensitivity.

In conclusion, berberine and nandinine inhibited IKK $\beta$ activation with beneficial regulation of adipokine production and improved insulin-mediated glucose uptake in an AMPK-dependent manner in adipocytes. Although, some studies report that a hy-

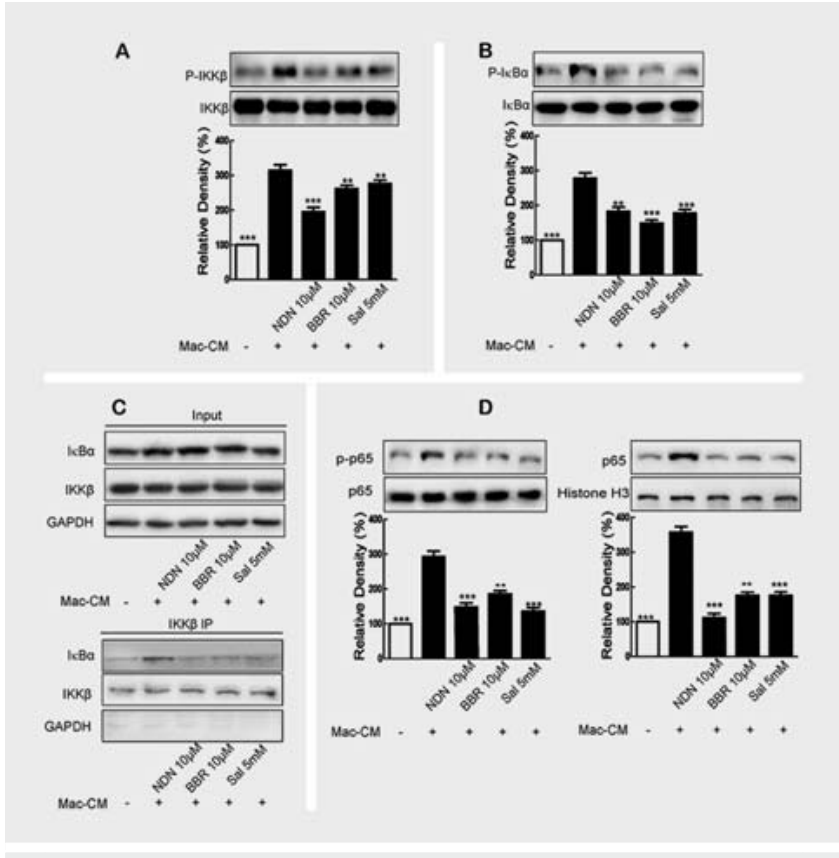

- Fig. 3 Berberine and nandinine inhibited IKK $\beta / N F-K B$ activation in adipocytes. Cells were pretreated with berberine $(10 \mu \mathrm{M})$, nandinine $(10 \mu \mathrm{M})$, or salicylate $(5 \mathrm{mM})$ for $1 \mathrm{~h}$ and then stimulated with Mac-CM for $30 \mathrm{~min}$. Phosphorylation of IKK $\beta$, IKB $\alpha$, and p65 ( $A$ and $B)$, and expression of p65 in the nuclear fraction (D) were determined by Western blot analysis. The interaction of IKK $\beta$ and $I K B \alpha$ was detected by immunoprecipitation (C). Results are expressed as the mean $\pm S D$ of three independent experiments. ${ }^{*} P<0.01$ vs. Mac-CM, ${ }^{* * *} \mathrm{p}<0.001$ vs. Mac-CM. Mac-CM, group with Mac-CM treatment alone; NDN, nandinine; BBR, berberine; Sal, salicylate.

droxyl group of flavonoids affects oral anti-inflammatory activity. In our study, the anti-inflammation effect of nandinine was similar to that of berberine both in vivo and in vitro. Perhaps other factors also determine the anti-inflammatory effects of nandinine. Overall, our results reinforced the scientific basis underlying the use of berberine and nandinine in the implementation of functional food and supported the development of novel therapeutic products.

\section{Materials and Methods}

\section{Chemicals and biochemicals}

Lipopolysaccharide (Escherichia coli serotype 055:B5, LPS), compound C (purity $\geq 98 \%$ by HPLC analysis), protein A/G PLUS-Agarose, AICAR (purity $\geq 98 \%$ by HPLC analysis), and insulin were obtained from Sigma-Aldrich. Anti-IRS-1 (R301; BS1408); anti-Phospho-IRS-1 (Ser307; BS4725), anti-Akt (A444; BS1810), anti-Phospho-Akt (T308; BS4008), anti-IKK $\beta$ (F182; BS1407), anti-PhosphoIKK $\beta$ (Y199; BS4320), anti-Na+/K+-ATPase $\alpha-1$ (BS1436), GAPDH (AP0063), HRP-conjugated anti-rabbit, and anti-mouse lgG antibodies were purchased from Bioworld Technology. PY99 (sc7020) was purchased from Santa Cruz Biotechnology. Histone $\mathrm{H} 3$, anti-AMPK $\alpha$, anti-phospho-AMPK $\alpha$ (T172), and anti-GLUT-4 were purchased from Cell Signaling Technology. 2-NBD-glucose (N13195, lot: 873337) was purchased from Invitrogen. Sodium 


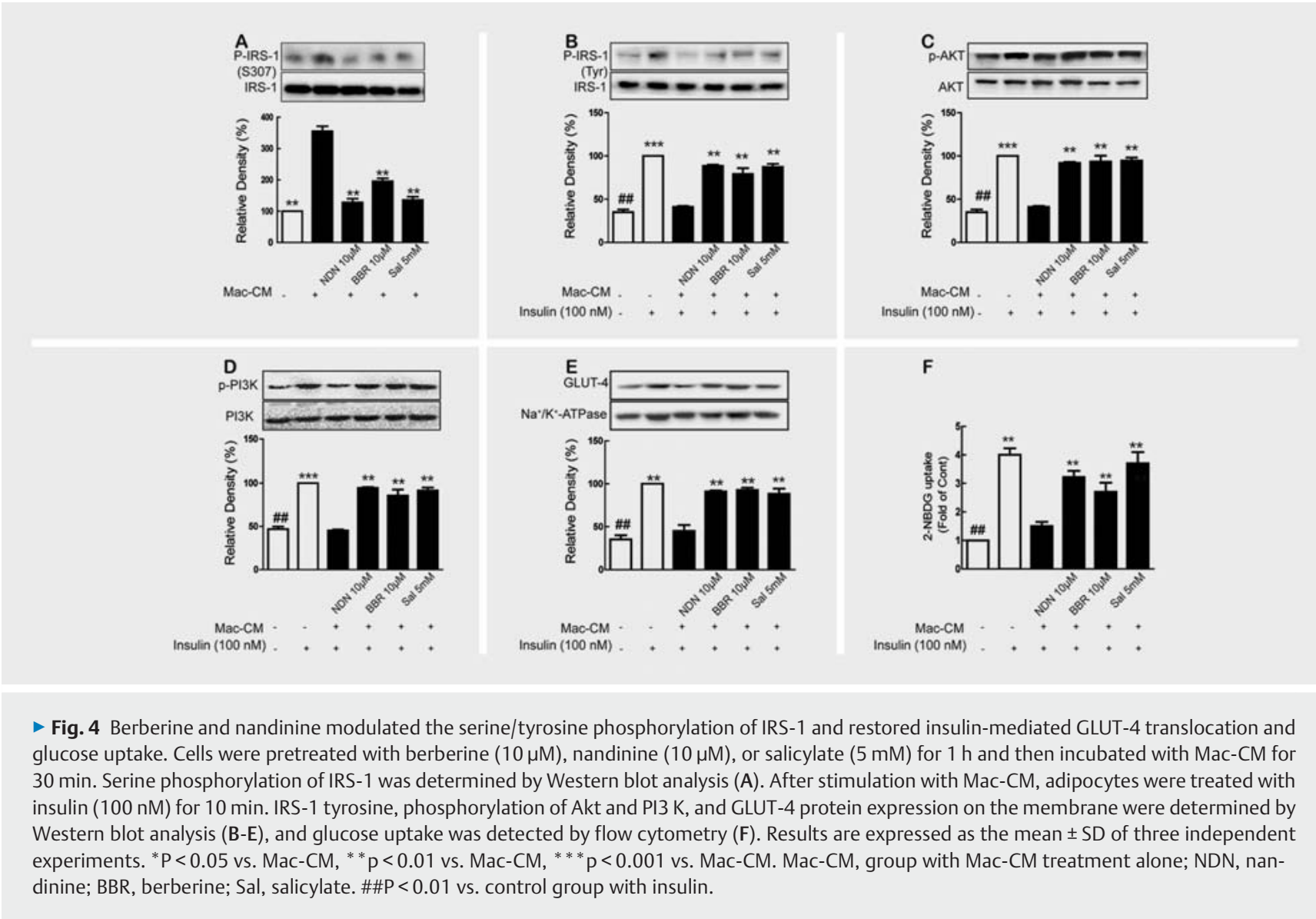

salicylate (purity $\geq 99.5 \%$ ) was purchased from Tianjin Kemiou Chemical Agent Center. Fraction-PREP Cell Fractionation Kit was purchased from BioVision.

\section{Animals}

The male ICR mice (6-8 weeks of age), used all throughout the experiments, were supplied by the Laboratory Animal Center of Nanjing Qinglongshan. The care and treatment of these mice were maintained in accordance with the Provisions and General Recommendation of Chinese Experimental Animals Administration Legislation. The animal protocol was approved by Animal Ethics Committee of School of Chinese Materia Medica, China Pharmaceutical University (Permission Number: 2012-2-20) and the exact date of approval was February 25, 2012.

\section{Plant material}

Berberine (Kunming Fengshanjian Medical Research Co., Ltd.; purity $>98 \%$ ) and nandinine (a gift from Dr. Zhang Jian, Department of Complex Prescription of TCM, China Pharmaceutical University; purity $>98 \%$ ) were dissolved in DMSO, and the final concentration of DMSO in the medium or water was $<0.1 \%$. The structures are shown in $~$ Fig. 1. Salicylate (purity $\geq 99.5 \%$ ) was a product of Tianjin Kemiou Chemical Agent Center.

\section{Preparation of macrophages-derived conditioned medium}

Mice were killed by cervical dislocation and injected intraperitoneally with $5 \mathrm{ml}$ of PBS. After gentle abdominal massage, the PBS containing peritoneal macrophages was collected and cultured in 6 -well plates $\left(2 \times 10^{6} /\right.$ well $)$ for $2 \mathrm{~h}$. Adherent cells were cultured in serum-free DMEM and stimulated by $5 \mu \mathrm{g} / \mathrm{ml}$ lipopolysaccharide (LPS) for $24 \mathrm{~h}$. The supernatant was collected by centrifuge at $4{ }^{\circ} \mathrm{C}$ as a macrophage-derived medium (Mac-CM). TNF- $\alpha$ and IL- 6 in the supernatant were measured with ELISA kits (R\&D). When levels of TNF- $\alpha$ and IL- 6 were greatly elevated compared with the control, the macrophages were activated. Mac-CM was filtered through a $0.22-\mu \mathrm{m}$ filter and stored at $-70^{\circ} \mathrm{C}$.

\section{T3-L1 Cell culture and differentiation}

The 3T3-L1 cell line, which is a cell line of preadipocytes, was obtained from the cell bank of the Chinese Academy of Sciences, and cultured in DMEM with $10 \% \mathrm{FBS}$ at $37^{\circ} \mathrm{C}$ in a $5 \% \mathrm{CO}_{2}$ atmosphere until cells grew to confluence. At 2 days after full confluence, cells were differentiated via incubation in DMEM containing $0.5 \mathrm{mM}$ isobutylmethylxanthine (IBMX), $1 \mu \mathrm{m}$ dexamethasone, $10 \mu \mathrm{g} / \mathrm{ml}$ insulin for $48 \mathrm{~h}$, and then for 2 days in DMEM (10\% FBS) containing $10 \mu \mathrm{g} / \mathrm{ml}$ insulin alone. Cells were maintained and refed every 2 days with DMEM, $25 \mathrm{mM}$ glucose, and $10 \%$ FBS. After 8-12 days, over $80 \%$ of the cells exhibited the adipocyte phenotype with large lipid droplets in the cytoplasm. 
A

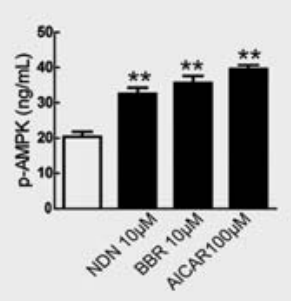

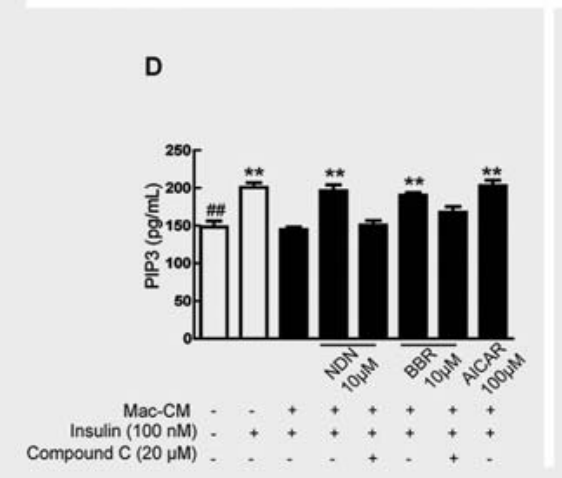

B

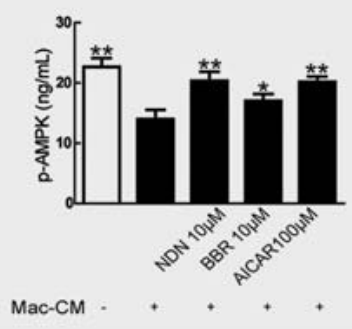

C

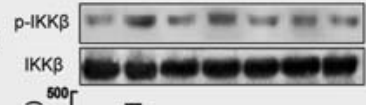

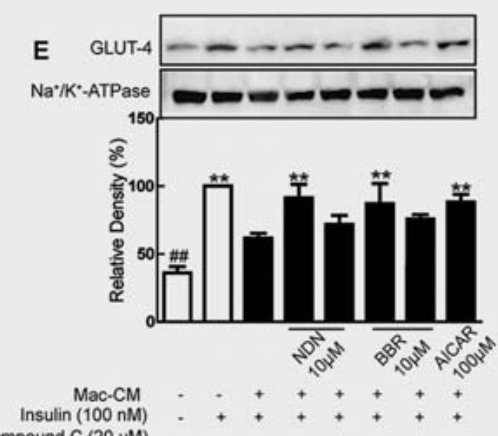

Compound $\mathrm{C}(20 \mu \mathrm{M})$

\begin{abstract}
- Fig. 5 Berberine and nandinine affected AMPK activation to inhibit inflammation and regulated insulin resistance in adipocytes. Adipocytes were treated with berberine $(10 \mu \mathrm{M})$, nandinine $(10 \mu \mathrm{M})$, or AICAR $(100 \mu \mathrm{M})$ for $1 \mathrm{~h}$. The activity of AMPK was determined with an ELISA kit (A). The result is expressed as the mean $\pm S D(n=3)$. ${ }^{*} P<0.01$ vs. control group. Adipocytes were pretreated with berberine $(10 \mu M)$, nandinine $(10 \mu M)$, compound C $(20 \mu \mathrm{M})$, or AICAR $(100 \mu \mathrm{M})$ for $1 \mathrm{~h}$. Then, they were incubated with Mac-CM for 30 min followed or not by a 10-min exposure to insulin $(100 \mathrm{nM})$. The activity of AMPK was determined with an ELISA kit (B). IKK $\beta$ phosphorylation was determined by Western blot analysis (C). Production of PIP3 was determined by ELISA (D). Membrane protein was extracted and GLUT-4 content was determined by Western blot analysis (E). Before insulin was added, the 2-NBDG probe $(10 \mu \mathrm{M})$ was added, and cells were incubated at $37^{\circ} \mathrm{C}$ for $40 \mathrm{~min}$. Glucose uptake was detected with fluorescence by flow cytometry $(F)$. The results are expressed as the mean $\pm S D(n=3)$. ${ }^{* *} \mathrm{P}<0.01 \mathrm{vs}$. Mac-CM. Mac-CM, group with Mac-CM treatment alone; \#\#p<0.01 vs. control group with insulin. NDN, nandinine; BBR, berberine; Sal, salicylate.
\end{abstract}

\section{Determination of TNF- $\alpha$, IL-6, and adiponectin production in adipocytes}

Cells were plated in 24-well cell culture plates at a density of $2 \times 10^{5}$ cells/well and fasted for $24 \mathrm{~h}$. Cells were pretreated with tested agents at determined concentrations for $1 \mathrm{~h}$ and then incubated in diluted Mac-CM $(1: 1, v / v)$ for $24 \mathrm{~h}$. TNF- $\alpha$, IL-6, and adiponectin in the medium were quantified with an ELISA kit (eBioscience).

\section{AMPK and PI3K activity assay in adipocytes}

Adipocytes $\left(1 \times 10^{6}\right.$ per well) were incubated for $4 \mathrm{~h}$ in serum-free DMEM pretreated with berberine $(10 \mu \mathrm{M})$, nandinine $(10 \mu \mathrm{M})$, compound C $(20 \mu \mathrm{M})$, or AICAR $(100 \mu \mathrm{M})$ for $1 \mathrm{~h}$. Subsequently, these adipocytes were exposed to Mac-CM for another $30 \mathrm{~min}$ followed or not by a 10 -min treatment of insulin $(100 \mathrm{nM})$. After washing with ice-cold PBS, cells were lysed in cell lysis buffer (Tris- $\mathrm{HCl} 50 \mathrm{mM}, \mathrm{pH} 7.2$, containing $1 \%$ sodium deoxycholate, $0.1 \%$ sodium dodecyl sulfate, $1 \% \mathrm{NP}-40, \mathrm{NaCl} 0.15 \mathrm{M}$, sodium orthovanadate $1 \mathrm{mM}$ ). The lysates were centrifuged at $13000 \mathrm{~g}$ for $40 \mathrm{~min}$ at $4{ }^{\circ} \mathrm{C}$. The supernatant was collected for the AMPK activity assay or for the quantification of PIP3, the product of PI3K with ELISA Kits (Dizhao Biotech), respectively.

\section{Western blotting}

Cells were collected and lysed with the sample buffer $(62.5 \mathrm{mM}$ Tris- $\mathrm{HCl}, \mathrm{pH} 6.8,2 \%$ sodium dodecyl sulfate, $10 \%$ glycerol, $50 \mathrm{mM}$ dithiothreitol, $0.1 \%$ bromophenol blue). Total proteins (30-50 $\mathrm{gg}$ ) were resolved by $10 \%$ SDS-PAGE and then transferred electrophoretically to PVDF membranes. After blocking and washing, the membranes were detected by chemiluminescence (ECL system) and exposed by autoradiography, followed by immunoblotting with first and second antibodies.

\section{2-(N-[7-Nitrobenz-2-oxa-1,3-diazol-4-yl] amino)-2- deoxyglucose assay}

To analyze glucose uptake, the fluorescent glucose analog 2-(N[7-nitrobenz-2-oxa-1,3-diazol-4-yl] amino)-2-deoxyglucose (2NBDG), which allows for direct quantification of glucose incorporation in living cells by flow cytometry, was used [35]. After related pretreatment, cells were incubated with the NBDG probe $(10 \mu \mathrm{M})$ for $40 \mathrm{~min}$ and insulin $(100 \mathrm{nM})$ for $10 \mathrm{~min}$. Then, cells were washed with PBS thrice, and fluorescence density was measured by a flow cytometry using a FACSCanto ${ }^{\mathrm{TM}}$ system (BD Biosciences). 


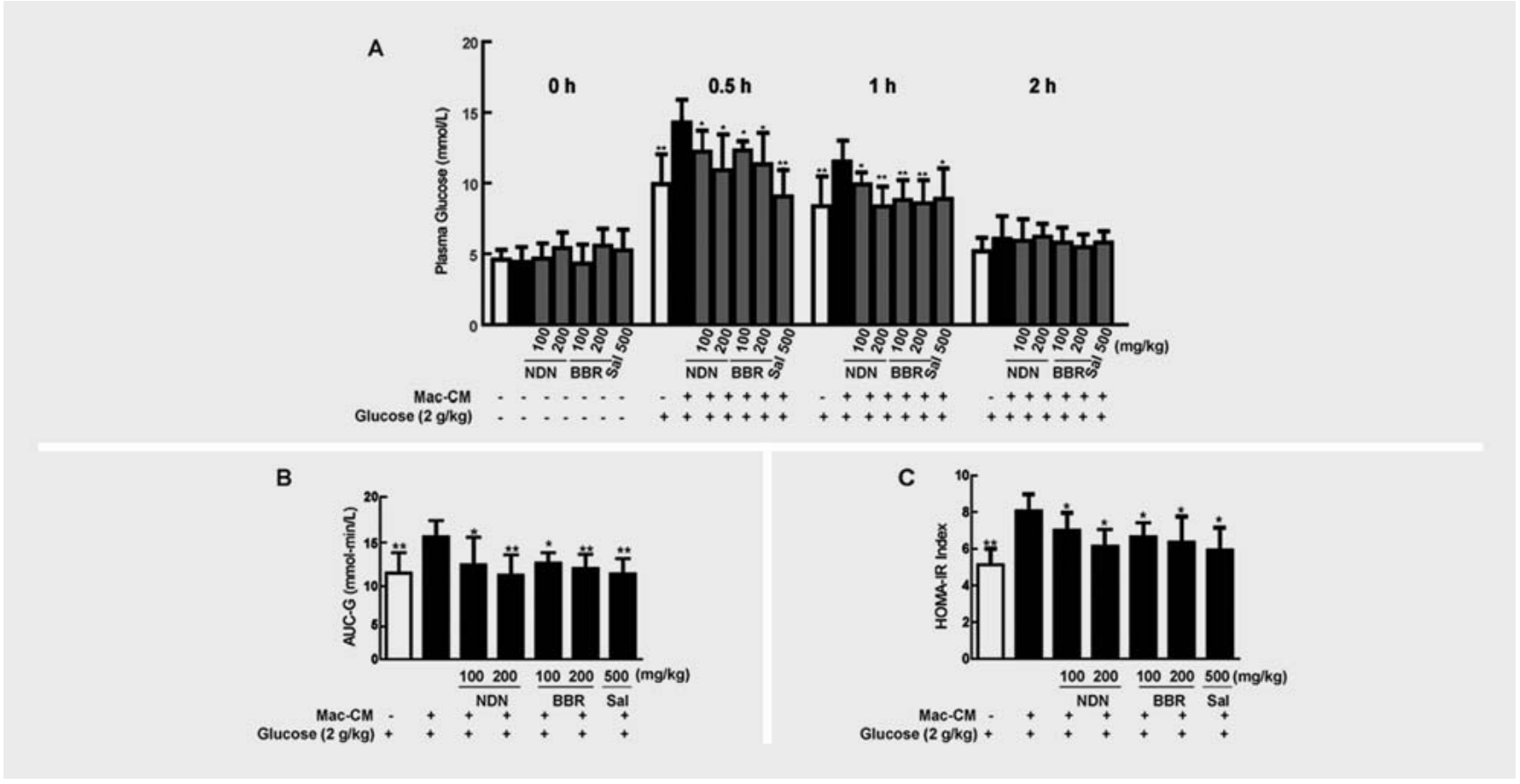

- Fig. 6 Berberine and nandinine reversed glucose intolerance and enhanced insulin sensitivity in vivo. Overnight-fasted mice were given nandinine (100 and $200 \mathrm{mg} / \mathrm{kg}$ ), berberine (100 and $200 \mathrm{mg} / \mathrm{kg})$, or salicylate $(500 \mathrm{mg} / \mathrm{kg})$ by gavage. After $1 \mathrm{~h}$, Mac-CM $(0.1 \mathrm{~mL} / 10 \mathrm{~g}$, diluted with saline, $1: 1, \mathrm{v} / \mathrm{v})$ was intraperitoneally injected followed by oral administration of glucose $(2 \mathrm{~g} / \mathrm{kg})$ after another $30 \mathrm{~min}$. Blood was collected from the orbital sinus at regular intervals after the glucose load. A Glucose concentrations within $2 \mathrm{~h}$ after the glucose load. B AUC-G, area under curve for glucose. C Homeostasis model assessment of the insulin resistance (HOMA-IR) index. The results are expressed as the mean $\pm S D(n=8) ;{ }^{*} p<0.05$ vs. Mac-CM, ${ }^{*}{ }^{*} \mathrm{p}<0.01$ vs. Mac-CM, ${ }^{* * *} \mathrm{p}<0.001$ vs. Mac-CM. Mac-CM, group with Mac-CM treatment alone; NDN, nandinine; BBR, berberine; Sal, salicylate.

\section{Real-time PCR}

Total RNA was extracted using TRIzol reagent. Quantitative realtime PCR (RT-PCR) was performed using SYBR Green PCR reagents. The specific primers for TNF- $\alpha$ were $5^{\prime}$-CTGTAGCCCACGTCGTAGC-3' (forward) and 5'-CTGTAGCCCACGTCGTAGC-3' (reverse). For IL-6, the primers were $5^{\prime}$-TCCAGTTGCCTTCTTGGGAC-3' (forward) and 5' - GTGTAATTAAGCCTCCGACTTG-3' (reverse). For adiponectin, the primers were $5^{\prime}$-TGTTCCTCTTAATCCTGCCCA-3' (forward) and 5'-CCAACCTGCACAAGTTCCC TT-3' (reverse). For GAPDH, the primers were $5^{\prime}$-CATGACCACAGTCCATGCCATCAC-3' (forward) and 5'-TGAGGTCCACCACCCTGTTGCTGT-3' (reverse). Briefly, $C D N A(1 \mu \mathrm{L})$ from the RT reaction was added to $10 \mu \mathrm{L}$ of the RT-PCR mixture containing $5 \mu \mathrm{L}$ of Master Mix and $0.2 \mu \mathrm{M}$ forward and reverse primers. The samples were incubated at $95^{\circ} \mathrm{C}$ for $2 \mathrm{~min}$, followed by 40 cycles at $95^{\circ} \mathrm{C}$ for $15 \mathrm{~s}$ and at $60^{\circ} \mathrm{C}$ for $1 \mathrm{~min}$. The samples were assessed by $2^{-\Delta \Delta \mathrm{ct}}$ relative quantitative analysis to determine the expression differences.

\section{Immunoprecipitation}

After determination of the protein concentrations, the cell extract was incubated with anti-IKK $\beta$ antibody $(2 \mu \mathrm{g})$ for $2 \mathrm{~h}$ at $4{ }^{\circ} \mathrm{C}$ and then incubated with $20 \mu \mathrm{L}$ of protein $\mathrm{A} / \mathrm{G}$ plus-agarose beads overnight with constant shaking. Afterward, the beads were washed thrice with ice-cold radio immunoprecipitation assay buffer. The bound protein was extracted by adding $40 \mu \mathrm{L}$ of $2 \times$ SDS sample buffer and boiling for $5 \mathrm{~min}$. The complexes were subjected to SDS-PAGE followed by Western blot.

\section{Preparation of nuclear fractions}

The nuclear proteins were isolated using the Fraction-PREP Cell Fractionation Kit according to the manufacturer's instructions.

\section{Glucose and insulin tolerance test in mice}

Mice were fasted for $12 \mathrm{~h}$ and orally administrated berberine, nandinine, or salicylate at the given concentrations. After $1 \mathrm{~h}$, mice were intraperitoneally injected with $0.2 \mathrm{ml}$ of diluted macrophages-CM $(1: 2, v / v)$. After $30 \mathrm{~min}$, mice were orally administered glucose solution $(2 \mathrm{~g} / \mathrm{kg})$. Blood was collected from the orbital sinus at indicated times. Blood glucose was determined with a commercial kit based on the glucose oxidase peroxidase (GOD-POD) method. To determine insulin tolerance, mice were administered insulin $(0.5 \mathrm{U} / \mathrm{kg}$, s.c) $30 \mathrm{~min}$ after Mac-CM treatment. AUC (AUC-G) for blood glucose was calculated as follow: $0.5 \times[\mathrm{Bg} 0+\mathrm{Bg} 30] / 2+0.5 \times[\mathrm{Bg} 30+\mathrm{Bg} 60] / 2+1 \times[\mathrm{Bg} 60+$ $\mathrm{Bg} 120] / 2$ (Bg, blood glucose). To determine insulin sensitivity, blood was collected 30 min after oral glucose load, and blood glucose and insulin concentrations were measured simultaneously (ELISA kit for insulin assay). Homeostasis model assessment of the insulin resistance (HOMA-IR) index was calculated according to the following formula: HOMA-IR index = blood glucose (mmol/ L) $\times$ blood insulin $(\mathrm{mlU} / \mathrm{L}) / 22.5[36]$. 


\section{Statistical analysis}

Data were expressed as means \pm SD. Differences between groups were analyzed using Prism 5.0 (GraphPad Software Inc.), and statistical analysis was performed using one-way ANOVA, followed by the Student-Newman-Keuls test. A value of $p<0.05$ was considered statistically significant.

\section{Acknowledgements}

This work is supported by the National Natural Science Foundation of China (Grant No. 81072976) and the National Nature Science Foundation of China (NSFC NO. 21302052). Our department is partly supported by the project funded by the Priority Academic Program Development of Jiangsu Higher Education Institutions.

\section{Conflict of Interest}

The authors declare no conflict of interest.

\section{References}

[1] Heilbronn LK, Campbell LV. Adipose tissue macrophages, low grade inflammation and insulin resistance in human obesity. Curr Pharm Des 2008; 14: $1225-1230$

[2] Gregor MF, Hotamisligil GS. Inflammatory mechanisms in obesity. Annu Rev Immunol 2011; 29: 415-445

[3] Coppack SW. Pro-inflammatory cytokines and adipose tissue. Proc Nutr Soc 2001; 60: 349-356

[4] Fain JN. Release of interleukins and other inflammatory cytokines by human adipose tissue is enhanced in obesity and primarily due to the nonfat cells. Vitam Horm 2006; 74: 443-477

[5] De Luca C, Olefsky JM. Inflammation and insulin resistance. FEBS Lett 2008; 582: 97-105

[6] Rasouli N, Kern PA. Adipocytokines and the metabolic complications of obesity. J Clin Endocrinol Metab 2008; 93: S64-S73

[7] Towler MC, Hardie DG. AMP-activated protein kinase in metabolic control and insulin signaling. Circ Res 2007; 100: 328-341

[8] Yang Z, Kahn BB, Shi H, Xue BZ. Macrophage alpha1 AMP-activated protein kinase (alpha1AMPK) antagonizes fatty acid-induced inflammation through SIRT1. J Biol Chem 2010; 285: 19051-19059

[9] Shoelson SE, Lee J, Goldfine AB. Inflammation and insulin resistance. I Clin Invest 2006; 116: 1793-1801

[10] Piyanuch R, Sukhthankar M, Wandee G, Baek SJ. Berberine, a natural isoquinoline alkaloid, induces NAG-1 and ATF3 expression in human colorectal cancer cells. Cancer Lett 2007; 258: 230-240

[11] Zhou H, Mineshita S. The effect of berberine chloride on experimental colitis in rats in vivo and in vitro. J Pharmacol Exp Ther 2000; 294: 822-829

[12] Godugu C, Patel AR, Doddapaneni R, Somagoni J, Singh M. Approaches to improve the oral bioavailability and effects of novel anticancer drugs berberine and betulinic acid. PLoS One 2014; 9: e89919

[13] Chen W, Miao YQ, Fan D], Yang SS, Lin X, Meng LK, Tang X. Bioavailability study of berberine and the enhancing effects of TPGS on intestinal absorption in rats. AAPS PharmSciTech 2011; 12: 705-711

[14] Ouchi N, Walsh K. Adiponectin as an anti-inflammatory factor. Clin Chim Acta 2007; 380: 24-30

[15] Ouchi N, Parker JL, Lugus JJ, Walsh K. Adipokines in inflammation and metabolic disease. Nat Rev Immunol 2011; 11: 85-97

[16] Hoesel B, Schmid JA. The complexity of NF-kappaB signaling in inflammation and cancer. Mol Cancer 2013; 12: 86-100
[17] Baker RG, Hayden MS, Ghosh S. NF-kappaB, inflammation, and metabolic disease. Cell Metab 2011; 13: 11-22

[18] Lee H], Joo M, Abdolrasulnia R, Young DG, Choi I, Ware LB, Blackwell TS, Christman BW. Peptidylarginine deiminase 2 suppresses inhibitory \{kappa]B kinase activity in lipopolysaccharide-stimulated RAW 264.7 macrophages. J Biol Chem 2010; 285: 39655-39662

[19] Li ], Houseknecht KL, Stenbit AE, Katz EB, Charron MJ. Reduced glucose uptake precedes insulin signaling defects in adipocytes from heterozygous GLUT4 knockout mice. FASEB J 2000; 14: 1117-1125

[20] Chang L, Chiang SH, Saltiel AR. Insulin signaling and the regulation of glucose transport. Mol Med 2004; 10: 65-71

[21] Bastard JP, Maachi M, Lagathu C, Kim M], Caron M, Vidal H, Capeau ], Feve $B$. Recent advances in the relationship between obesity, inflammation, and insulin resistance. Eur Cytokine Netw 2006; 17: 4-12

[22] Xu H, Barnes GT, Yang Q, Tan G, Yang D, Chou C], Sole J, Nichols A, Ross JS, Tartaglia LA, Chen $\mathrm{H}$. Chronic inflammation in fat plays a crucial role in the development of obesity-related insulin resistance. J Clin Invest 2003; 112: 1821-1830

[23] Johnson AR, Milner J], Makowski L. The inflammation highway: metabolism accelerates inflammatory traffic in obesity. Immunol Rev 2012; 249: 218-238

[24] Qatanani M, Lazar MA. Mechanisms of obesity-associated insulin resistance: many choices on the menu. Genes Dev 2007; 21: 1443-1455

[25] Faubert B, Boily G, Izreig S, Griss T, Samborska B, Dong ZF, Dupuy F, Chambers C, Fuerth BJ, Viollet B, Mamer OA, Avizonis D, DeBerardinis RJ, Siegel PM, Jones RG. AMPK is a negative regulator of the Warburg effect and suppresses tumor growth in vivo. Cell Metab 2013; 17: 113-124

[26] Salt IP, Palmer TM. Exploiting the anti-inflammatory effects of AMP-activated protein kinase activation. Expert Opin Investig Drugs 2012; 21 : 1155-1167

[27] Ou HC, Lee W], Wu CM, Chen JF, Sheu WH. Aspirin prevents resistin-induced endothelial dysfunction by modulating AMPK, ROS, and Akt/ eNOS signaling. J Vasc Surg 2012; 55: 1104-1115

[28] Lihn AS, Pedersen SB, Richelsen B. Adiponectin: action, regulation and association to insulin sensitivity. Obes Rev 2005; 6: 13-21

[29] Benomar Y, Gertler A, De Lacy P, Crepin D, Ould Hamouda H, Riffault L, Taouis $M$. Central resistin overexposure induces insulin resistance through Toll-like receptor 4. Diabetes 2013; 62: 102-114

[30] Herschkovitz A, Liu YF, Ilan E, Ronen D, Boura-Halfon S, Zick Y. Common inhibitory serine sites phosphorylated by IRS-1 kinases, triggered by insulin and inducers of insulin resistance. J Biol Chem 2007; 282: 1801818027

[31] Salminen A, Kaarniranta K. AMP-activated protein kinase (AMPK) controls the aging process via an integrated signaling network. Ageing Res Rev 2012; 11:230-241

[32] Abel ED, O'Shea KM, Ramasamy R. Insulin resistance: metabolic mechanisms and consequences in the heart. Arterioscler Thromb Vasc Biol 2012; 32: 2068-2076

[33] Jeong HW, Hsu KC, Lee JW, Ham M, Huh JY, Shin HJ, Kim WS, Kim JB. Berberine suppresses proinflammatory responses through AMPK activation in macrophages. Am J Physiol Endocrinol Metab 2009; 296: E955E964

[34] Wilcox G. Insulin and insulin resistance. Clin Biochem Rev 2005; 26: 19-39

[35] Sukumar M, Liu J, Ji Y, Subramanian M, Crompton JG, Yu Z, Roychoudhuri R, Palmer DC, Muranski P, Karoly ED, Mohney RP, Klebanoff CA, Lal A, Finkel T, Restifo NP, Gattinoni L. Inhibiting glycolytic metabolism enhances CD8+ T cell memory and antitumor function. J Clin Invest 2013; 123: 4479-4488

[36] Salama RM, Schaalan MF, Elkoussi AA, Khalifa AE. Potential utility of sodium selenate as an adjunct to metformin in treating type II diabetes mellitus in rats: a perspective on protein tyrosine phosphatase. Biomed Res Int 2013; 2013: 231378 